\title{
ISOSPECTRAL CONVEX DOMAINS IN THE HYPERBOLIC PLANE
}

\author{
CAROLYN S. GORDON AND DAVID L. WEBB
}

(Communicated by Peter Li)

\begin{abstract}
We construct pairs of nonisometric convex polygons in the hyperbolic plane for which the Laplacians are both Dirichlet and Neumann isospectral. We also give examples of pairs of isospectral potentials for the Schrödinger operator on certain convex hyperbolic polygons.
\end{abstract}

Given a bounded domain $\Omega$ with piecewise-smooth boundary in a Riemannian manifold $M$, denote by $\operatorname{Spec}_{\mathrm{D}}(\Omega)$ (respectively, $\operatorname{Spec}_{\mathrm{N}}(\Omega)$ ) the eigenvalue spectrum of the Laplace-Beltrami operator acting on smooth functions on $\Omega$ with Dirichlet (respectively, Neumann) boundary conditions. A pair of domains $\Omega_{1}$ and $\Omega_{2}$ in $M$ is Dirichlet isospectral if $\operatorname{Spec}_{\mathrm{D}}\left(\Omega_{1}\right)=\operatorname{Spec}_{\mathrm{D}}\left(\Omega_{2}\right)$; Neumann isospectrality is similarly defined.

Mark Kac's question "Can one hear the shape of a drum?" [K] asks whether Dirichlet isospectral domains in the Euclidean plane must be isometric. Recently, the authors and Wolpert [GWW1, 2] answered Kac's question negatively by exhibiting a pair of nonisometric domains in the Euclidean plane which are Dirichlet and Neumann isospectral; the construction also yields isospectral domains in the round 2-sphere and in the hyperbolic plane. Using similar methods, Buser et al. [BCDS] constructed other examples. In all cases, however, the domains are nonconvex. Thus Kac's question for convex plane domains remains open.

The purpose of this note is to exhibit pairs of convex domains in the hyperbolic plane which are both Dirichlet and Neumann isospectral. These domains are obtained from those of [GWW1, 2] by modifying the shape of the fundamental tile used in the construction, as in [BCDS]; Bérard's extension [B1] of Sunada's Theorem [S] facilitates the proof of isospectrality by "transplantation" of eigenfunctions from one domain to the other, as in [BCDS, B2].

Let $T$ be a hyperbolic triangle with vertex angles $\alpha, \beta$, and $\gamma$, and let $\Omega_{\alpha, \beta, \gamma}$ be the hyperbolic polygon composed of seven copies of the tile $T$ glued together as in Figure 1 on the next page; whenever two triangles share an edge, each is the reflection of the other about their common edge. The domain depicted in Figure 1 is constructed from the triangle with angles $\alpha=\gamma=\pi / 3$, $\beta=\pi / 4$, which results in a convex quadrilateral; other choices of angles lead to other polygons. Figure 2 depicts $\Omega_{\pi / 4, \pi / 4, \pi / 3}$ and $\Omega_{\pi / 3, \pi / 4, \pi / 4}$.

Received by the editors July 6, 1992.

1991 Mathematics Subject Classification. Primary 58G25; Secondary 53C20.

Both authors gratefully acknowledge partial support from NSF grants. 


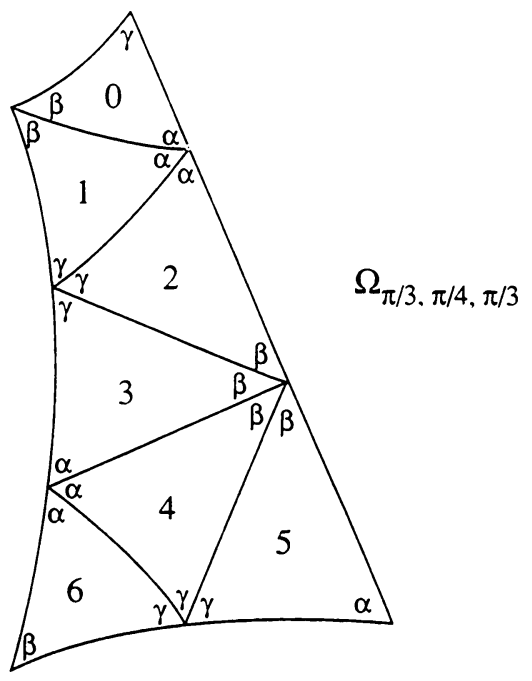

FIGURE 1

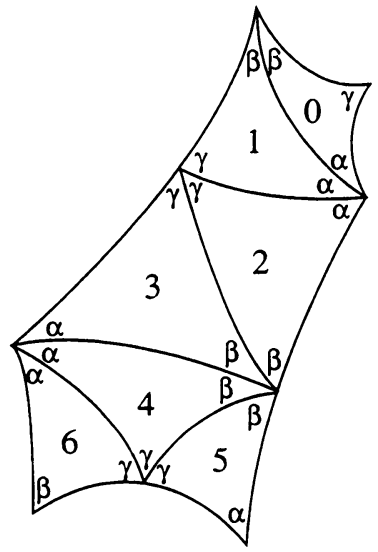

$\Omega_{\pi / 4, \pi / 4, \pi / 3}$

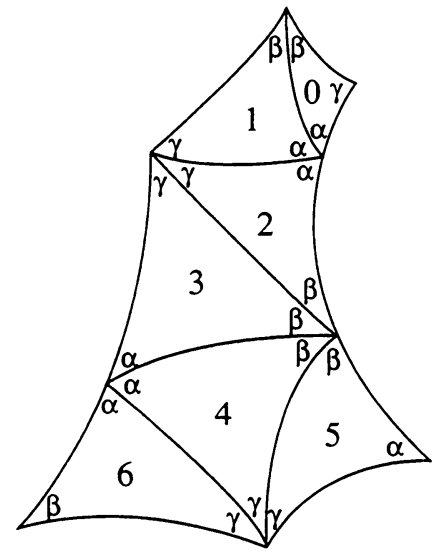

$\Omega_{\pi / 3, \pi / 4, \pi / 4}$

FIGURE 2

Theorem. Let $\alpha, \gamma \leq \pi / 3$ and $\beta \leq \pi / 4$. Then the hyperbolic domains $\Omega_{\alpha, \beta, \gamma}$ and $\Omega_{\gamma, \beta, \alpha}$ are convex polygons which are both Dirichlet and Neumann isospectral. They are isometric if and only if $\alpha=\gamma$.

Proof. If $\alpha=\gamma$, then the domains are easily seen to be isometric. Conversely, if $\alpha$ and $\gamma$ are unequal, then a comparison of the ordering of the vertex angles as one traverses the boundaries of the two domains shows that $\Omega_{\alpha, \beta, \gamma}$ and $\Omega_{\gamma, \beta, \alpha}$ are not isometric.

The inequalities on $\alpha, \beta$, and $\gamma$ obviously ensure convexity of the domains.

Before proving isospectrality, we note that the same combinatorial gluing pattern was used with a different shaped fundamental tile in [GWW1, 2] and [BCDS] to produce nonconvex isospectral plane domains. The isospectrality proof is the same; we briefly review it. The proof uses Berard's extension 
of Sunada's Theorem to provide an explicit "transplantation" isomorphism which carries a $\lambda$-eigenfunction on $\Omega_{\alpha, \beta, \gamma}$ to a $\lambda$-eigenfunction on $\Omega_{\gamma, \beta, \alpha}$, as follows. Given $f \in L^{2}\left(\Omega_{\alpha, \beta, \gamma}\right)$, let $f_{0}, f_{1}, \ldots, f_{6}$ be the restrictions of $f$ to the tiles labelled $0,1, \ldots, 6$ in $\Omega_{\alpha, \beta, \gamma}$ (see Figure 1 ). Let $[f]=$ ${ }^{t}\left(f_{0}, f_{1}, \ldots, f_{6}\right)$, a column vector of functions on $T$. Let $S_{\mathrm{D}}$ be the matrix

$$
\left[\begin{array}{rrrrrrr}
a & -a & a & -b & b & -a & -b \\
-a & a & -b & a & -a & b & b \\
a & -b & a & -a & b & -b & -a \\
-b & a & -a & b & -a & b & a \\
b & -a & b & -a & b & -a & -a \\
-a & b & -b & b & -a & a & a \\
-b & b & -a & a & -a & a & b
\end{array}\right]
$$

and let $S_{\mathrm{N}}$ be the same matrix with all minus signs replaced by plus signs, where $a$ and $b$ are chosen so that $S_{\mathrm{D}}$ and $S_{\mathrm{N}}$ are orthogonal. Then the transplantation of Dirichlet eigenfunctions sends an eigenfunction $f$ on $\Omega_{\alpha, \beta, \gamma}$ to the function $g$ on $\Omega_{\gamma, \beta, \alpha}$ determined by $[g]=S_{\mathrm{D}}[f]$. One easily verifies that $g$ is a Dirichlet eigenfunction on $\Omega_{\gamma, \beta, \alpha}$ by inspection. The Neumann transplantation is defined similarly, using $S_{\mathrm{N}}$.

Remark. When $\alpha=\gamma$, a construction analogous to that in [GWW2, §5] produces pairs of isospectral potentials for the Schrödinger operator on $\Omega_{\alpha, \beta, \gamma}$. In particular, for $\alpha=\gamma=\pi / 3$ and $\beta=\pi / 4$ as in Figure 1, this yields isospectral Schrödinger operators on a convex quadrilateral in the hyperbolic plane.

\section{REFERENCES}

[B1] P. Bérard, Transplantation et isospectralite. I, Math. Ann. 292 (1992), 547-559.

[B2] Domaines plans isospectraux a la Gordon-Webb-Wolpert: une preuve terre a terre, preprint.

[BCDS] P. Buser, J. H. Conway, P. Doyle, and K.-D. Semmler, Some planar isospectral domains, preprint.

[GWW1] C. Gordon, D. Webb, and S. Wolpert, One cannot hear the shape of a drum, Bull. Amer. Math. Soc. (N.S.) 27 (1992), 134-138.

[GWW2] __ Isospectral plane domains and surfaces via Riemannian orbifolds, Invent. Math. 110 (1992), 1-22.

[K] M. Kac, Can one hear the shape of a drum?, Amer. Math. Monthly 73 (1966), 1-23.

[S] T. Sunada, Riemannian coverings and isospectral manifolds, Ann. of Math. (2) 121 (1985), 169-186.

[U] H. Urakawa, Bounded domains which are isospectral but not congruent, Ann. Sci. École Norm. Sup. (4) 15 (1982), 441-456.

Department of Mathematics, Dartmouth College, Hanover, New Hampshire 03755

E-mail address: carolyn.s.gordon@dartmouth.edu

E-mail address: david.1. vebbedartmouth.edu 